\title{
Variational Segmentation Framework in Prolate Spheroidal Coordinates for 3D Real-Time Echocardiography
}

\author{
Bruno Vallet ${ }^{\mathrm{a}}$, Elsa D. Angelini ${ }^{\mathrm{b}}$, Andrew F. Laine ${ }^{\mathrm{a}}$ \\ ${ }^{\mathrm{a}}$ Department of Biomedical Engineering, Columbia University, ET351, 1210 Amsterdam Avenue, \\ New York, NY, 10027, USA; \\ ${ }^{\mathrm{b}}$ Département Traitement du Signal et des Images, CNRS UMR 5141 LTCI, Ecole Nationale \\ Supérieure des Télécommunications (ENST), GET, 46 rue Barrault, 75013 Paris, France;
}

\begin{abstract}
This paper presents a new formulation of a deformable model segmentation in prolate spheroidal coordinates for segmentation of 3D cardiac echocardiography data. The prolate spheroidal coordinate system enables a representation of the segmented surface with descriptors specifically adapted to the "ellipsoidal" shape of the ventricle. A simple data energy term, based on gray-level information, guides the segmentation. The segmentation framework provides a very fast and simple algorithm to evolve an initial ellipsoidal object towards the endocardial surface of the myocardium with near real-time deformations. With near real-time performance, additional constraints on landmark points, can be used interactively to prevent leakage of the surface.
\end{abstract}

Keywords: Real-time three-dimensional ultrasound, deformable model, finite element model, prolate spheroidal coordinates, deformable model

\section{INTRODUCTION}

We are interested in the class of segmentation methods based on deformable models. Physically-based deformable models, referred to as snake, are based on elasticity theory. Lagrangian equations of non-rigid motion are expressed in terms of position functions in Euclidean 3D space. The deformable model representation is parametric and the segmentation algorithm locates each point in space as a function of time. Deformation of the model is applied on its surface via minimization of individual equations of motion for each Cartesian coordinates. This energy expresses the equilibrium of internal and external forces on the surface. Such deformable models were introduced by Kass et al. ${ }^{1}$ as 2D explicit deformable contours and generalized to the 3D case by Terzopoulos et al. ${ }^{2}$. A second family of segmentation methods based on deformable models was introduced by Osher and Sethian ${ }^{3}$ These models are based on an implicit formulation of the surface to deform, embedded in a level set function.

In an original approach, Caselles et al. ${ }^{4}$ proposed a reformulation of the snake deformable model as the definition of a geodesic curve whose length is constrained by image-based data information. For these three families of deformable models, initial data force relied on image gradients while regularization of the curve was achieved by constraining the motion of the curve with its curvature.

A separate family of segmentation methods was proposed by Mumford and Shah ${ }^{5}$ to segment an image into smooth areas and a "finite" set of contours. This variational approach was reformulated recently by Chan and Vese ${ }^{6}$ into a level-set like deformable model approach where the data force measures the homogeneity of the inside volume enclosed by the deforming surface.

The level set implementation for this family of geometric deformable models increases by 1 the dimensionality of the minimization space and relies on volume integrals for the data term.

Segmentation of echocardiographic data with level-set based deformable models has been proposed by several groups, mainly on 2D ultrasound data. We cite as an example Paragios ${ }^{7}$ who proposed a shape-driven variational segmentation approach, with temporal tracking of the image data via optical flow and use of prior statistical models of gray level

Medical Imaging 2006: Image Processing, edited by Joseph M. Reinhardt, Josien P. W. Pluim, Proc. of SPIE Vol. 6144, 61444A, (2006) · 0277-786X/06/\$15 - doi: 10.1117/12.653692 
value distributions of the myocardium, the blood pool and the surrounding tissues. In a more recent study, Sarti et al. ${ }^{8}$ proposed a maximum likelihood segmentation method implemented in a level-set framework with Rayleigh probability distribution models.

Different parameterization schemes of the endocardial surfaces were proposed to simplify the tasks of segmentation, tracking and deformation characterizations. Stetten et al. ${ }^{9}$ proposed a fuzzy labeling segmentation method which used a radial representation of the ventricular shape, based on hand-drawing training shapes with cylindrical symmetries. They applied their method to 3D ultrasound data. Jacob et al. ${ }^{10}$ proposed a spatio-temporal deformable model framework based on the parameterization of 2D endocardial contours with B-splines and using 1D intensity-invariant phase-based image features. Shape-space basis functions were defined from a set of training manually segmented contours, leading to a time-consuming learning process.

The idea developed in this paper follows a similar paradigm, trying to significantly reduce the complexity of the manipulation of a deformable model by describing the segmentation surface with a limited number of shape parameters. To do so we need to adapt the parameterization of the segmentation surface according to the geometry of the objects to segment. In our case, being interested in the segmentation of 3D echocardiographic data to extract the ventricular cavities, we opted for a prolate spheroidal coordinate system. The choice of this coordinate system was motivated by the cardiac modeling framework proposed by Hunter ${ }^{11}$.

\section{METHOD}

\section{Segmentation surface description}

We consider our image data on a domain $\Omega$ in $\mathbb{R}^{3}$. The segmentation task is modeled as the partition of the space $\Omega$ into two domains (inside and outside of the surface) separated by the boundary of the closed surface $K$ in $\Omega$.

\section{Coordinate system}

In his approach, Hunter proposed a coordinate system in which the representation of the myocardium requires a minimal set of parameters. As the geometry of the heart ventricles resembles an ellipsoid, Hunter used a coordinate system called prolate spheroidal. This coordinate system is illustrated in defined as:

$$
\begin{aligned}
& x=\sinh (\lambda) \cos (\theta) \sin (\mu) \\
& y=\sinh (\lambda) \sin (\theta) \sin (\mu) \\
& z=\cosh (\lambda) \cos (\mu)
\end{aligned}
$$

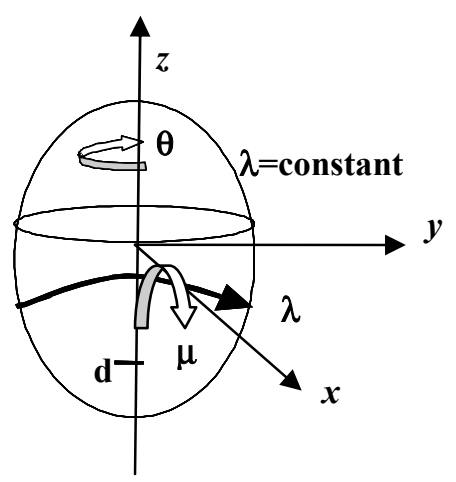

Figure 1: Prolate spheroidal coordinate system.

In this coordinate system, isosurfaces with $\lambda$ constant are ellipsoids. Even though we focus in this paper on prolate spheroidal coordinates, we can also consider in this work cylindrical and spherical coordinate systems and for this 
reason, we will use a general formulation of the coordinate system for parameterization of the deformable surface: $\left(v_{0}, v_{1}, v_{2}\right)$. The 3 general variables of the $3 \mathrm{D}$ coordinate system relate to the specific coordinate systems mentioned above as follows:

$$
\begin{aligned}
& \left(v_{0}, v_{1}, v_{2}\right)=(r, \theta, z) \text { in cylindrical coordinates } \\
& \left(v_{0}, v_{1}, v_{2}\right)=(r, \theta, \varphi) \text { in spherical coordinates } \\
& \left(v_{0}, v_{1}, v_{2}\right)=(\lambda, \theta, \mu) \text { in prolate spheroidal coordinates }
\end{aligned}
$$

In these coordinate systems, it is convenient to describe the surface through an equation of the form:

$$
v_{0}=f_{\text {ref }}\left(v_{1}, v_{2}\right)
$$

Hunter's descriptions used this representation to describe ventricular shapes, where $f_{\text {ref }}$ are bicubic Hermite interpolation functions.

\section{Hermite interpolation}

\subsection{D case}

In $1 \mathrm{D}$, let $f_{\text {ref }}(v):\left[v_{\min } v_{\text {max }}\right] \rightarrow \mathbb{R}$ be a reference function for which we know the values $H_{r e f}^{0, i}=f_{\text {ref }}\left(v_{i}\right)$

and derivatives $H_{r e f}^{1, i}=\frac{d f_{r e f}\left(v_{i}\right)}{d x}$ on $N$ points:

$$
v_{i}=v_{\min }+\left(v_{\max }-v_{\min }\right) \frac{i}{N}, i=\{0,1, \ldots, N\}
$$

The cubic Hermite interpolation of $f_{\text {ref }}(v)$ is defined on patches $P_{i}=\left[v_{i} v_{i+1}\right]$ by:

$$
f_{r e f}(v)=H_{r e f}^{0, i} \psi_{0}^{0}(V)+H_{r e f}^{1, i} \psi_{0}^{1}(V)+H_{r e f}^{0, i+1} \psi_{1}^{0}(V)+H_{r e f}^{1, i+1} \psi_{1}^{1}(V)
$$

or in more general terms:

$$
f_{r e f}(v)=\sum_{d=0,1} \sum_{\delta i=0,1} H_{r e f}^{d, i+\delta i} \psi_{\delta i}^{d}(V)
$$

where $v \in\left[v_{i} v_{i+1}\right]$ and $V=\left(v-v_{i}\right) /\left(v_{i+1}-v_{i}\right)=N\left(v-v_{i}\right) /\left(v_{\max }-v_{\min }\right)$ is the local coordinate of point $v$. The functions $\psi_{\delta i}^{d}(V)$ are the Hermite basis functions, defined as: 


$$
\begin{cases}\psi_{0}^{0}(\mathrm{~V})=1-3 \mathrm{~V}^{2}+2 \mathrm{~V}^{3} \quad \psi_{0}^{0^{\prime}}(\mathrm{V})=6 \mathrm{~V}(\mathrm{~V}-1) \\ \psi_{0}^{1}(\mathrm{~V})=\mathrm{V}(\mathrm{V}-1)^{2} \quad \psi_{0}^{1^{\prime}}(\mathrm{V})=(3 \mathrm{~V}-1)(\mathrm{V}-1) \\ \psi_{1}^{0}(\mathrm{~V})=\mathrm{V}^{2}(3-2 V) \quad \psi_{1}^{0^{\prime}}(\mathrm{V})=6 \mathrm{~V}(1-\mathrm{V}) \\ \psi_{1}^{1}(\mathrm{~V})=\mathrm{V}^{2}(V-1) \quad \psi_{1}^{1^{\prime}}(\mathrm{V})=\mathrm{V}(3 \mathrm{~V}-2)\end{cases}
$$

From the definition of the Hermite interpolation functions we have: $\psi_{i}^{j}(V) \times \psi_{k}^{l}(V)=\delta_{i k}(V) \delta_{j l}(V)$, which ensures that they form a basis of the real axis, on which the $H_{r e f}^{d, i}$, called the Hermite coefficients, constitute the coordinates in this basis.

We point out here that if $\psi_{\delta i}^{d}(V)$ are called the Hermite basis functions, they are not exactly the basis functions referenced in the decomposition of the function $f_{\text {ref }}$. These basis functions are:

$$
\begin{cases}f_{i}^{d}(V)=\psi_{1}^{d}(V), & \text { if } v \in\left[v_{i-1} v_{i}\right] \\ f_{i}^{d}(V)=\psi_{0}^{d}(V), & \text { if } v \in\left[v_{i} v_{i+1}\right] \\ 0 \text { elsewhere } & \end{cases}
$$

With these basis functions we can write the standard decomposition of $f_{\text {ref }}$ as:

$$
f_{\text {ref }}(\mathrm{v})=\sum_{\mathrm{i}=0}^{\mathrm{N}} \sum_{\mathrm{d}=0}^{1} H_{r e f}^{d, i} f_{i}^{d}(v)
$$

The $f_{i}^{d}$ basis functions are translated versions of the function $f_{0}^{d}$, plotted in Figure 2.
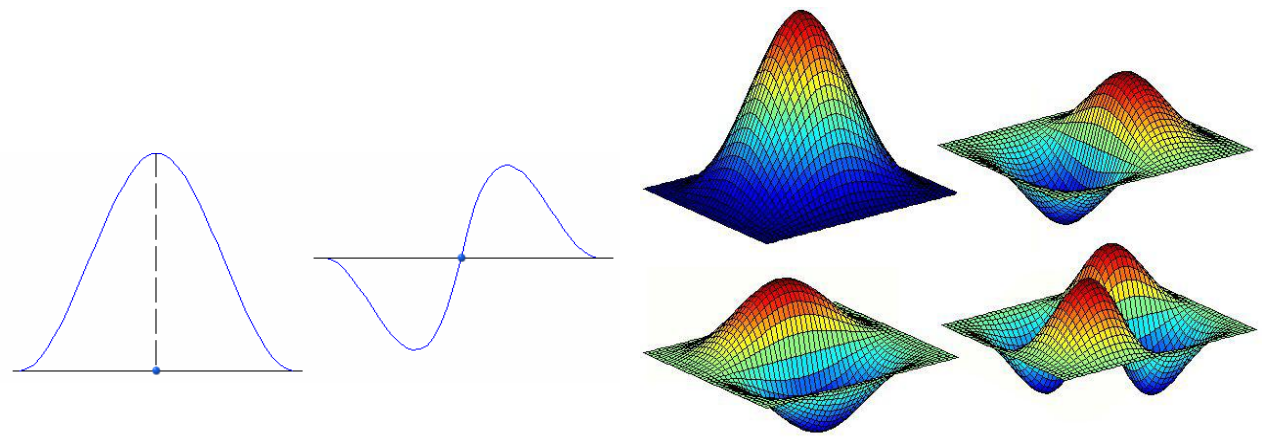

Figure 2: Hermite basis functions in 1D (left) and 2D (right).

For numerical implementation, we normalized these basis functions for the $L^{2}$ norm using:

$$
\left|f_{i}^{0}\right|^{2}=\frac{26}{35} \quad\left|f_{i}^{1}\right|^{2}=\frac{2}{105}
$$




\subsection{D case}

In $2 \mathrm{D}$, we decompose the function $f_{\text {ref }}\left(v_{1}, v_{2}\right):\left[\begin{array}{ll}v_{1}^{\min } & v_{1}^{\max }\end{array}\right] \times\left[v_{2}^{\min } v_{2}^{\max }\right] \rightarrow \mathbb{R}^{2}$ for which we know the values of the function, its derivatives and cross derivatives: $H_{r e f}^{0,0, i_{1}, i_{2}}, H_{r e f}^{1,0, i_{1}, i_{2}}, H_{r e f}^{0,1, i_{1}, i_{2}}, H_{r e f}^{1,1, i_{1}, i_{2}}$ at points $\left(v_{1}^{i_{1}}, v_{2}^{i_{2}}\right)$ with: $v_{k}^{i_{k}}=v_{k}^{\min }+\left(v_{k}^{\max }-v_{k}^{\min }\right) \frac{i_{k}}{N_{k}}, i_{k}=0,1, \ldots, N_{k}$

The parameter $N_{k}$ represents the number of discretization points in the $k^{\text {th }}$ direction. By interpolating each of the Hermite coefficients in Equation (6), we get a 2D interpolation of $f_{\text {ref }}$ on $P_{i, j}=\left[v_{1}^{i} v_{1}^{i+1}\right] \times\left[v_{2}^{j} v_{2}^{j+1}\right]$ by:

$$
f_{\text {ref }}\left(v_{1}, v_{2}\right)=\sum_{d_{1}=0}^{1} \sum_{d_{2}=0}^{1} \sum_{i_{1}=0}^{N_{1}} \sum_{i_{2}=0}^{N_{2}} H_{r e f}^{d_{1}, d_{2}, i_{1}, i_{2}} f_{i_{1}, i_{2}}^{d_{1}, d_{2}}\left(v_{1}, v_{2}\right)
$$

where

$$
f_{i_{1}, i_{2}}^{d_{1}, d_{2}}\left(v_{1}, v_{2}\right)=f_{i_{1}}^{d_{1}}\left(v_{1}\right) f_{i_{2}}^{d_{2}}\left(v_{2}\right)
$$

and the local coordinates of the components in (8) for $v_{k} \in\left[v_{k}^{i} v_{k}^{i+1}\right]$ are:

$$
V_{k}=\frac{\left(v_{k}-v_{k}^{i}\right)}{\left(v_{k}^{i+1}-v_{k}^{i}\right)}=\frac{N_{k}\left(v_{k}-v_{k}^{i}\right)}{\left(v_{k}^{\max }-v_{k}^{\min }\right)}
$$

Here, the basis functions are translated versions of the 4 basis functions $f_{0,0}^{d_{1}, d_{2}}$ represented in Figure 2.

By interpolating the $f_{\text {ref }}$ function, we get a description of the segmentation surface $K$ given by $v_{0}=f_{\text {ref }}\left(v_{1}, v_{2}\right)$ using only the $4 \times N_{1} \times N_{2}$ parameters $H_{r e f}^{d_{1}, d_{2}, i_{1}, i_{2}}$ (which we will call $H_{r e f}^{i}$ in the remaining of this paper, as indices do not matter). From a practical point of view, values for $N_{k}$ are usually lower than 10, and Hunter even suggested using $N_{1}=N_{2}=4$ for cardiac ventricles. This allows us to describe a segmented ventricular surface with less than 400 values to update, independently of the data size. For comparison, 3D level set methods require 1 computation per data voxel, leading to a computational space size up to $10^{6}$ for echocardiographic data with standard spatial resolution.

\section{Segmentation}

The object of this section is to derive a simple segmentation framework using a separation surface $K\left(H_{r e f}^{i}\right)$. Data information at node points $V$ will be extracted and input into a data force to deform the surface via modification of the $H_{r e f}^{i}$ coefficients that describe $K$. 


\subsection{Minimal partition problem}

For a general formulation of the deformable model segmentation framework, we chose the Mumford and Shah variational approach restricted to the segmentation of the image data $u_{0}$ into a partition $\Omega_{i}$ of $\Omega$ of piecewise constant functions.

This problem is called the "minimal partition problem" and is formulated as the minimization of the following functional:

$$
\inf _{c_{i}, k}\left\{F_{M S}\left(c_{i}, K\right)=\sum_{i} \int_{\Omega_{i}}\left|u_{0}-c_{i}\right|^{2}+v \int_{K} d H^{1}\right\}
$$

The term $H^{1}$ represents the length of the contour $K$ and the minimum of the energy is reached when $c_{i}$ is the average value of $u_{0}$ inside $\Omega_{i}$.

This problem was formulated into a level set framework by Chan and Vese in ${ }^{6}$ with volume integrals. In a recent work, Jehan-Besson et al. derived a minimization approach for region-based variational segmentation approaches using shape gradients ${ }^{12}$. In this work, we are looking at the problem of minimizing the functional $F_{M S}$ according to the descriptors $H_{r e f}^{i}$ of $K$.

\subsection{Application to graph equation surfaces}

We call $\vec{x}\left(v_{0}, v_{1}, v_{2}\right)$ the $(x, y, z)$ Cartesian coordinates of a point, function of its $v_{i}$ variables in a given coordinate system. We now want to segment the data with a separation surface $K\left(H_{r e f}^{i}\right)$ described by a graph equation, i.e. under the form $v_{0}=f_{\text {ref }}\left(v_{1}, v_{2}\right)$.

Hence a point of the surface is parameterized by $\vec{x}_{K}\left(v_{0}, v_{1}, v_{2}\right)=\vec{x}_{K}\left(f_{\text {ref }}\left(v_{1}, v_{2}\right), v_{1}, v_{2}\right)$ where $f_{\text {ref }}$ is the function describing $K$ and parameterized by the $H_{r e f}^{i}$ coefficients.

For a given coordinate system, we define the following entities:

- The Jacobian vectors:

$\vec{J}_{i}=\left[\frac{\partial \vec{x}}{\partial v_{i}}\right]=h_{i} \vec{u}_{i}$

and the Jacobian matrix

$J=\left[\vec{J}_{1} \vec{J}_{2} \ldots \vec{J}_{N}\right]$

- The scale factors

$h_{i}=\left\|\vec{J}_{i}\right\|=\sqrt{\sum_{j=1}^{n}\left(\frac{\partial x_{j}}{\partial v_{i}}\right)^{2}}$.

- The local basis 
$\overrightarrow{u_{i}}=\frac{\vec{J}_{i}}{\left\|\vec{J}_{i}\right\|}=\frac{\partial \vec{x}\left(v_{0}, v_{1}, v_{2}\right)}{h_{i} \partial v_{i}}$

These definitions allow us to give an expression of the elementary surface area $d A_{K}$ of the contour $K$ and its elementary inside volume $d V_{K}$ as:

- Elementary surface area $d A_{K}$ :

with

$$
d A_{K}=A_{K} d v_{1} d v_{2}
$$

$$
A_{K}=\left\|\frac{\partial \vec{x}}{\partial v_{1}} \wedge \frac{\partial \vec{x}}{\partial v_{2}}\right\|=\left\|\left(f_{r e f}^{1} \overrightarrow{J_{0}}+\overrightarrow{J_{1}}\right) \wedge\left(f_{r e f}^{2} \overrightarrow{J_{0}}+\overrightarrow{J_{2}}\right)\right\|
$$

where

$$
f_{r e f}^{i}=\frac{\partial f_{r e f}}{\partial v_{i}}
$$

and

$$
\begin{aligned}
A_{K} & =\left\|h_{1} h_{2} \overrightarrow{u_{0}}-f_{r e f}^{1} h_{0} h_{2} \overrightarrow{u_{1}}-f_{r e f}^{2} h_{0} h_{1} \overrightarrow{u_{2}}\right\| \\
& =\sqrt{\left(h_{1} h_{2}\right)^{2}+\left(f_{r e f}^{1} h_{0} h_{2}\right)^{2}+\left(f_{r e f}^{2} h_{0} h_{1}\right)^{2}}
\end{aligned}
$$

-Elementary volume $d V_{K}$ :

$$
d V_{K}=\operatorname{Det}(J) d v_{0} d v_{1} d v_{2}
$$

with

$$
\operatorname{Det}(J)=\left|\vec{J}_{1} \cdot\left(\vec{J}_{2} \wedge \vec{J}_{3}\right)\right|
$$

and therefore

$$
\begin{aligned}
d V_{K} & =h_{0} h_{1} h_{2} d v_{0} d v_{1} d v_{2} \\
& =V_{K} d v_{0} d v_{1} d v_{2}
\end{aligned}
$$

We are now going to look for a computation strategy for the $H_{r e f}^{i}$ coefficients of the surface $K$ in order to achieve a good segmentation of the data. Going back to the deformable model segmentation framework, energy minimization usually requires to be able to compute the gradient of the energy to minimize. In our case, computation of the first order derivatives of $F_{M S}$ in $H_{r e f}^{i}$ requires to first re-write the Mumford-Shah function $F_{M S}$ in equation (14) with a Heaviside function $\mathrm{H}$ as:

$$
F_{M S}\left(c_{i}, K\right)=\int_{\Omega}\left(\left|u_{0}-c_{\text {in }}\right|^{2} H+\left|u_{0}-c_{\text {out }}\right|^{2}(1-H)\right)+v \int_{K} d H^{1}
$$


where the Heaviside $H$ function is defined as:

$$
H\left(v_{0}, v_{1}, v_{2}\right)=\left\{\begin{array}{ll}
1 & \text { if } v_{0} \leq f_{\text {ref }}\left(v_{1}, v_{2}\right) \\
0 & \text { otherwise }
\end{array} .\right.
$$

With the formulation of the image data term in $F_{M S}$ as a volume integral, we compute:

$$
\frac{\partial F_{M S}}{\partial H_{r e f}^{i}}=\int_{v_{1}, v_{2}}\left(2 f_{i}\left(u_{0}-\frac{c_{\text {in }}+c_{\text {out }}}{2}\right)\left(c_{\text {out }}-c_{\text {in }}\right) V_{K}+v \frac{\partial A_{K}}{\partial H_{r e f}^{i}}\right) d v_{1} d v_{2}
$$

The quantities $\left(u_{0}, A_{K}, V_{K}, h_{i}\right)$ are $\Omega \rightarrow \mathbb{R}$ functions. In the integral, they are all being evaluated at the point $\vec{x}\left(f_{\text {ref }}\left(v_{1}, v_{2}\right), v_{1}, v_{2}\right)$.

The derivative of the surface term is computed as:

$$
\frac{\partial A_{K}}{\partial H_{r e f}^{i}}=h_{0}^{2} \frac{h_{2}^{2} f^{1} f_{i}^{1}+h_{1}^{2} f^{2} f_{i}^{2}}{A_{K}}+f_{i} \frac{\left(f^{1}\right)^{2} h_{2} h_{0}\left(h_{2} \dot{h}_{0}+h_{0} \dot{h}_{2}\right)+\left(f^{2}\right)^{2} h_{1} h_{0}\left(h_{1} \dot{h}_{0}+h_{0} \dot{h}_{1}\right)+h_{2} h_{1}\left(h_{2} \dot{h}_{1}+h_{1} \dot{h}_{2}\right)}{A_{K}}
$$

Since the $h_{i}$ are evaluated at $\left(v_{0}, v_{1}, v_{2}\right)$ and $v_{0}=f_{\text {ref }}\left(v_{1}, v_{2}\right)$ depends on $H_{\text {ref }}^{i}$, we evaluated the derivative of $h_{i}$ according to $H_{r e f}^{i}$ as:

$$
\frac{\partial h_{j}}{\partial H_{r e f}^{i}}=f_{i} \frac{\partial h_{j}}{\partial v_{0}}=f_{i} \dot{h}_{j}
$$

These equations may be used to solve the minimum partition problem with graph equation surfaces for any family of basis functions $f_{i}$ selected for the decomposition of $f_{\text {ref }}$.

\subsection{Numerical scheme}

Interestingly, the gradient of the energy $F_{M S}$ is much faster to compute than the energy itself, as the volume integral becomes a surface integral, thus winning an order of magnitude in computational complexity. This observation lead us to discard standard numerical optimization methods based on the opposite assumption and to only follow the gradient of the energy in our optimization process. Minimization of $F_{M S}$ was therefore performed with the very simple scheme:

$$
H_{r e f}^{i}((j+1) \Delta t)=H_{r e f}^{i}(j \Delta t)-\Delta t \frac{\partial F_{M S}(j \Delta t)}{\partial H_{r e f}^{i}}
$$

which defines the temporal iterative scheme updating the values of $H_{r e f}^{i}$, and the deformation of the segmentation surface $K\left(H_{r e f}^{i}\right)$. 


\section{RESULTS}

We present in this section some experiments for segmentation of real-time three-dimensional echocardiographic ultrasound data acquired with a SONOS 7500 scanner from Philips. The data set used for our experiments was of size [176 160 144] in Cartesian coordinates. We illustrate here the main concepts which emerged from these tests.
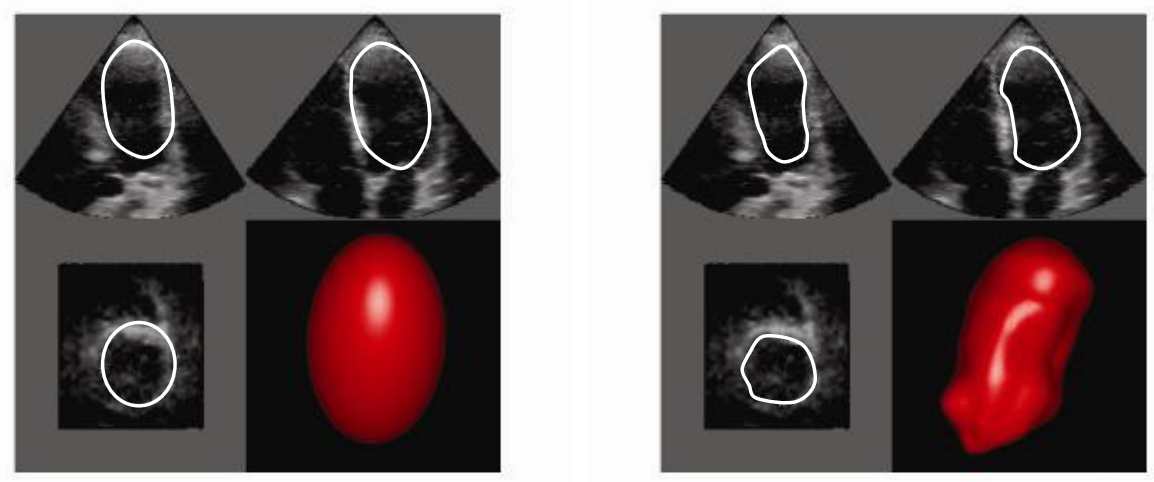

Figure 3: Segmentation of a 3D ultrasound volume with a prolate spheroidal surface representation. (left): Initial surface. (right): Segmented surface after 10 iterations.

\section{Influence of the parameters}

These experiments were performed with a surface representation in prolate spheroidal coordinates. Numerical implementation was ran with a discretization of the two angular parameters $(\theta, \mu)$ onto $(8,8)$ patches. To test computational speed limit of the method, the data force was simplified by manually setting the average value between the inside volume (i.e. the ventricular cavity) and the outside volume (i.e. the myocardium), which appears in Equation (22), to a fixed value, defined from the histogram of the data. The data force was evaluated on $8 \times 8$ points on each patch to combine the influence of the whole patch area on the increment of the radius value. Each patch element returned a radius increment that influenced the position of the neighboring nodes via interpolation with the basis functions.

The surface was initialized as an isosurface with $\lambda=1$. The scale factor and the orientation of the isosurface were defined from the manual selection of two points at the apex and the base of the ventricle. We list here a short discussion on the selection of numerical parameters:

- Time increment $\Delta t$ : The value of the time increment directly controls the increment of the radial coordinate and the volume of the segmented object. In our case, we found that a time increment $\Delta t=0.04$ corresponding to a $4 \%$ increment in radius and volume was well adapted.

- Number of iterations and stopping criteria: Fixing the interface gray value enabled to achieve a stable behavior of the segmentation process. About 10 iterations were required.

- Curvature term $v$ : The curvature term is negligible due to the intrinsic smoothness of the surface described in prolate spheroidal coordinates and interpolated by Hermite functions across a small number of patches. We can easily suppress this term without decreasing the quality of the segmentation while enhancing speed performance and simplicity. 


\section{Towards real time segmentation}

The complexity of the proposed algorithm does not depend on the size of the data, but only on the density of the discretization when evaluating the surface integrals in Equation (22). As data values are only needed on node points, the algorithm can be run with data given in any arbitrary geometry. This argument in especially interesting for 3D echocardiography where data is acquired in a spherical geometry. Our segmentation method could therefore be applied to the original data without the need for interpolation into Cartesian space. We performed some experiments for segmentation of 3D+Time data sets, with few iterations ( 3 to 5) between each frames, using the resulting surface as initialization for the next frame. With very few iterations, the first solutions were not correct, but the segmentation surface reached the heart walls in less than one heart beat, and then tracked the endocardial wall correctly over the next cycle. In this experiment, computation time between 2 frames got below $0.5 \mathrm{~s}$ on a standard desktop computer (Pentium IV $2.2 \mathrm{GHz}$ ) for a reasonable discretization of the surface. This very promising result allows us to believe that this algorithm may be implemented in real-time on $3 \mathrm{D}$ echocardiograph machines and could be used interactively by technicians and cardiologists. Finally, these surfaces can be very easily triangulated (using the integration points to build the triangles for instance), allowing fast and efficient 3D visualization.

\section{CONCLUSION}

We presented a formulation of the generic "variational" deformable model from Chan and Vese ${ }^{6}$ with a surface representation in prolate spheroidal coordinates that lead to near real-time segmentation, representing a leap of progress in performance when comparing to a standard level set implementations. Focusing on the segmentation of 3D echocardiographic data, our surface representation is particularly well adapted to the geometry of the ventricles, and the resulting surfaces are under a form widespread in the heart modeling community. In this respect, this method opens a new path in the assimilation of clinical data with anatomic modeling. From this point, many research directions might be explored, such as fitting more refine heart models with the data, or modifying the energies involved in the segmentation method for more accurate segmentation.

\section{BIBLIOGRAPHY}

1. $\quad$ M. Kass, A. Witkin and D. Terzopoulos, "Snakes: Active contour models." International Journal of Computer Vision, Vol. 1, pp. 321-331, 1987.

2. D. Terzopoulos and D. Metaxas, "Dynamic 3D models with local and global deformations:Deformable superquadratics." IEEE Transactions on Pattern Analysis and Machine Intelligence, Vol. 13, No. 7, pp. 703714, 1991.

3. S. Osher and J. A. Sethian, "Fronts propagating with curvature-dependent speed: Algorithms based on Hamilton-Jacobi formulations." Journal of Computational Physics, Vol. 79, No. 1, pp. 12-49, 1988.

4. V. Caselles, R. Kimmel and G. Sapiro, "Geodesic active contours." International Journal of Computer Vision, Vol. 22, pp. 61-79, 1997.

5. D. Mumford and J. Shah, "Boundary detection by minimizing functional", International Conference on Computer Vision and Pattern Recognition, pp. 22-26, San Francisco, CA, USA, 1985.

6. T. F. Chan and L. A. Vese, "Active contours without edges." IEEE Transactions on Image Processing, Vol. 10, No. 2, pp. 266 - 277, 2001.

7. $\quad$ N. Paragios, "A level set approach for shape-driven segmentation and tracking of the left ventricle." IEEE Transactions on Medical Imaging, Vol. 22, No. 6, pp. 773 -776, 2003.

8. A. Sarti, C. Corsi, E. Mazzini and C. Lamberti, "Maximum likelihood segmentation of ultrasound images with Rayleigh distribution." IEEE Transactions on Ultrasonics, Ferroelectrics and Frequency Control, Vol. 52, No. 6, pp. 947 - 960, 2005.

9. G. Stetten and S. Pizer, "Medial-Guided Fuzzy Segmentation", Medical Image Computing and Medical Computer-Assisted Intervention (MICCAI), Pittsburg, PA, USA, 2004.

10. G. Jacob, J. A. Noble, C. Behrenbruch, A. D. Kelion and A. P. Banning, "A shape-space-based approach to tracking myocardial borders and quantifying regional left-ventricular function applied in echocardiography." IEEE Transactions on Medical Imaging, Vol. 21, No. 3, pp. 226-238, 2002. 
11. P. J. Hunter and B. H. Smaill, "The analysis of cardiac function - a continuum approach." Progress in biophysics and molecular biology, Vol. 52, No. 2, pp. 101-164, 1988.

12. S. Jehan-Besson, M. Barlaud and G. Aubert, "DREAM2S: Deformable Region driven by an Euclidian Accurate Minimization Method for image and video segmentation." International Journal of Computer Vision, Vol. 53, No. 1, pp. 45-70, 2003. 